\title{
A case study: consolidation properties of Hazelwood Power Station ash
}

\author{
S Narendranathan GHD Pty Ltd, Australia
}

J Anders GHD Pty Ltd, Australia

J Faithful ENGIE, Australia

N Patel GHD Pty Ltd, Australia

\begin{abstract}
This paper presents the challenges involved in interpreting representative primary and secondary compression ratios within ash ponds at the Hazelwood brown coal mine, located in the Latrobe Valley region of Victoria. Rehabilitation of such ash ponds must include a suitably designed and robust capping liner. Unforeseen and excessive settlement can result in damage to the capping liner, diminishing its serviceability and impairing its longevity. This paper explores the process involved in the interpretation of compression ratios within ash, with a focus on the process utilised to refine the initial consolidation parameters with the observations from trial embankments. It is envisaged that by sharing this information with the wider mining fraternity that it will provide a useful precedent for industry practitioners involved in the assessment of brown coal ash.
\end{abstract}

Keywords: closure, consolidation, power station ash, brown coal, Asaoka method, mine rehabilitation

\section{Introduction}

The Hazelwood Power Station (Latrobe Valley, Victoria) is transitioning into closure and the authors are involved with the mine owners in assisting with the associated studies and assessments. One specific aspect that presents a unique challenge within brown coal mines is the decommissioning of ash ponds in such a manner that it does not burden future generations with adverse stability, environmental or social issues. Rehabilitation planning of Hazelwood Ash Pond No. 4 (HAP4) specifies the inclusion of a suitably designed capping liner. This capping liner must satisfy a number of stringent environmental and geotechnical criteria to remain serviceable into the future. A key geotechnical consideration is settlement of the underlying ash, which will occur over the life of the capping liner. In the case of HAP4 the underlying ash is several metres thick. Unforeseen differential and excessive settlement can result in damage to the capping liner, diminishing its serviceability and impairing its longevity.

Whilst it is common practice to undertake targeted borehole sampling and subsequent laboratory testing to characterise the consolidation properties of such materials, this process may not always yield definitive results on the scale of brown coal ash ponds, which in the case of HAP4 is on the order of 32 hectares. Should such an investigation programme be instituted, the inherent variability of the ash properties and sampling bias may render the results unrepresentative over a significant extent of the capping liner footprint. Further challenges arise when assessing settlement at HAP4 because, as far as the authors are aware, there is little relevant published work available in Australia. The most pertinent to this study being the work of Mudd et al. (2006), which investigated the consolidation properties of brown coal ash.

This paper presents a case study utilising HAP4 to demonstrate how the authors heuristically verified, with the benefit of trial embankments, the representativeness of laboratory-assessed ash consolidation parameters. Specifically, this paper articulates the challenges involved in interpreting representative primary and secondary compression ratios within the ash. As part of the field trials, the authors observed significant deviation in the back-calculated consolidation parameters from the trial embankment observations 
compared to those assessed analytically (in the laboratory) for similar ash material. The paper details the assessment methodology developed with a focus on the process utilised to refine the laboratory-assessed consolidation parameters with the observations from the trial embankments.

\section{Background}

When saturated soils (or mechanically similar materials) are subjected to an increase in vertical stress, there is an increase in the pore pressure. Dissipation of excess pore pressure is dependent upon the permeability of the material in question. The increased pore pressure dissipates much more slowly in clayey soils than in sandy soils. This pressure dissipation process involves the drainage of water from voids within the soil from areas of high hydraulic head to low hydraulic head. This ultimately leads to settlement consolidation of the soil. In Terzaghi (1943) developed a methodology that allowed the estimation of one-dimensional vertical consolidation of soil subjected to a change in the effective stress. Terzaghi's theory assumes a homogenous, fully saturated soil in which the soil particles and water are incompressible. Terzaghi's one-dimensional consolidation theory is commonly adopted in geotechnical engineering when estimating the magnitude of settlement of typical soils such as clay and sand. It is the authors' experience that the mechanical properties of brown coal ash differ somewhat from typical soils. As such, Terzaghi's one-dimensional consolidation theory may not, on its own, be reliable for interpreting time-settlement behaviour of the ash. Brown coal ash material cannot rely on the use of Terzaghi's theory alone; therefore an alternative approach has been developed by Asaoka (1978). This method has been adopted for this case study. Empirical methodologies such as that developed by Asaoka (1978) aim to quantify consolidation behaviour more accurately than when solely using Terzaghi's theory.

The benefit of using an empirical approach to determine consolidation properties of Victorian brown coal ash is that it can provide site specific information - i.e. settlement behaviour across a more representative and broader area of construction, as will be demonstrated in the case study. Asaoka's method involves graphically plotting the achievement of $90 \%$ primary consolidation to enable back calculation of the primary compression ratio. One benefit of Asaoka's method is that it does not require the data to be recorded until the achievement of 90 or $100 \%$ consolidation. Once a proper linear relationship is established, it is possible to interpret the end of primary consolidation; thereby facilitating the back calculation of primary compression coefficients.

Prevailing geotechnical characterisation processes involve the deployment of targeted investigation boreholes to retrieve suitable material samples for laboratory testing. As this process relies on the retrieval of 'point' samples it may not be representative of conditions over an extensive area Duncan (1993). Additionally, the very nature of intrusive material sampling renders the retrieval of suitable undisturbed samples challenging. This was a particular challenge at the Hazelwood ash ponds, as retrieval of suitable ash samples for laboratory testing is known to be inherently difficult, as samples are often disturbed or destroyed during sample recovery or test preparation. Accordingly, as is often the case with low strength and saturated materials, where samples are recovered, they tend to be indicative of the more competent ash material, and would unintentionally introduce a bias in relation to the consolidation properties. Should the traditional approach of sampling and testing be espoused for HAP4, it could result in the underestimation of final consolidation magnitudes and hence may underestimate the stain induced on the final capping liner.

\section{Investigation approach}

The overall aim was to obtain suitable and representative consolidation data across the footprint of HAP4. Considering the challenges already discussed, an observational approach after Asoka (1978) - which will be outlined in further detail-utilising trial pads to determine consolidation parameters across the underlying (in situ) ash was adopted. The benefits of employing this approach as opposed to a purely intrusive investigation campaign include:

- A trial embankment provides site specific performance information (settlement) across a representative and larger area of the capping liner footprint. In contrast, an investigatory campaign 
samples specific 'points', and may not adequately capture the spatial variability (both laterally and vertically) of the ash materials.

- A trial embankment is typically constructed on foundation materials, and the in situ state (e.g. moisture content and mechanical characteristics) of the foundation remain undisturbed. The process of intrusive drilling, sampling and preparation of test samples often disturbs, and can alter the mechanical characteristics of the obtained specimen. Such disturbance may affect the resulting consolidation parameters obtained from laboratory testing.

A chronology of the various phases of the case study is presented:

- Conceptual consolidation assessment - A desktop assessment that relied on ash consolidation/ compression parameters sourced from previous investigations of brown coal ash. The intent of this assessment was to provide an estimation of the anticipated settlements due to the construction of the capping liner for HAP4.

- Trial embankments and collection of settlement data - Based on the outcomes of the conceptual assessment, the locations of trial embankments were determined taking into consideration the variability of the ash and thickness across the HAP4 footprint and accessibility of earthworks plant to reach the proposed locations.

- Data interpretation - Survey instruments within the trial embankments were regularly surveyed to obtain absolute position and compute changes in instrument height. The data was interpreted using Asaoka's method (1978) to assess the achievement of primary compression. This enables the calculation of unique primary coefficients across the various ash types within HAP4.

- Calculation of settlements - Once primary and secondary compression parameters were obtained the magnitude of total settlements could be calculated across HAP4 and settlements predicted for the proposed capping liner design.

\section{$4 \quad$ Case study - Hazelwood Ash Pond No. 4 (HAP4)}

\subsection{Background and introduction to subject site}

HAP4 was constructed in 1982 as a settling pond for ash water from the Hazelwood Power Station. HAP4 was also used to store ash dredged from Hazelwood Ash Pond No. 1 (HAP1). A series of staged internal raises, using deposited ash, in 2007, 2012 and 2014, formed HAP4A within the main HAP4 embankments. Decant water was collected in the southeast corner of the pond and was returned to the power station for re-use via a return water pipeline. Ash disposal into HAP4 ceased in the first half of 2016.

The embankments are homogenous earthfill with a vertical chimney drain and horizontal blanket drain beneath the downstream shoulder. The western ridge of the site is formed by a natural hillside and the southern side abuts a decommissioned overburden dump referred to as the Eastern Overburden Dump (EOD). The original embankments were founded on alluvial soils and dumped overburden material. A clay blanket and stabilising berm were incorporated into the southern end of the embankment. Seepage through the embankments is collected in filter drains that discharge into a seepage collection system.

HAP4 has a surface area of approximately $320,000 \mathrm{~m}^{2}$ and, in its current condition, grades on the surface (from west to east) at less than 1\%, creating a large, essentially flat area. Ash deposition ceased in early 2016 in line with closure of the power station. HAP4 operates under an Environmental Protection Authority (EPA) Victoria Licence, where Schedule 2 specifies HAP4 is licensed to accept General Waste (Ashing Wastes).

\subsection{Regulatory framework and compliance requirements}

EPA Victoria (2015) Publication 788.3 Best Practice Environmental Management: Siting, Design, Operation and Rehabilitation of Landfills (Landfill BPEM) is the source document for best-practice siting, design, operation, performance and rehabilitation standards for landfills in Victoria. The design of the final cap for 
the HAP4 landfill has to address multiple elements to ensure cap performance, including material selection, seepage, settlement, liner stability, and water and leachate management in accordance with the Landfill BPEM.

A key element for landfill rehabilitation is capping design. The design objectives of the final HAP4 cap, as adopted from the Landfill BPEM, centres on minimising infiltration of water into the underlying material (ash), providing a long-term stable barrier between ash waste and the environment, and providing land suitable for its intended after-use. In short, the Landfill BPEM objective most pertinent to HAP4 is to ensure seepage of water into the landfill is minimised. Furthermore, the Landfill BPEM states that the impacts of settlement be considered as part of cap design, and the gradient for a completed cap should be sufficient to prevent water ponding on the cap to minimise seepage through the cap.

\subsection{HAP4 cap design}

To comply with the final cap gradients recommended in the Landfill BPEM and to facilitate drainage off the cap, the landfill cap surface for HAP4 was designed as a series of local ridges and gullies. It was recognised early in the design phase, that due to the sheer size of the site, importing fill to construct the subgrade to the cap would be impractical and cost prohibitive. As such, it was decided to work the deposited ash within HAP4 to form the subgrade in order to provide the stable foundation required for cap construction. The design has the worked ash surface mirroring the final cap surface in form and grades. To meet the required levels for the ash subgrade, considerable cut-to-fill earthworks will be required, which in-places will result in up to $8 \mathrm{~m}$ of ash fill being placed on top of the existing ash. Placement of the ash fill, and cap, will induce consolidation of the underlying in situ ash and overburden dump. Estimates indicate around $250,000 \mathrm{~m}^{3}$ of ash will go from cut to fill. However, the ability to accurately estimate cut-fill volumes is dependent on the amount of settlement that may occur across the site.

Consolidation of the underlying ash could compromise the cap gradients and final surface profile, which increases the potential for water seeping into the landfill. In addition, settlement increases strain on the capping, and the potential for cracking through the earthen layers of the barrier system, further increasing potential for seepage. Settlement was recognised as a key risk to the performance of the cap, and the ability of the cap design to meet the objectives of the Landfill BPEM.

\subsection{Ash properties and anticipated consolidation behaviour}

Based on the chronological depositional history of HAP4 as well as a number of past studies, the following is known in relation to the mechanical properties of the ash in HAP4 footprint (Figure 1):

- The sub-surface conditions are expected to consist generally of interbedded layers of ash (fine siltsize particles, within the delta) and charcoal (coarser sand-size particles, within the ash raise areas).

- Most of the material encountered is expected to be of soft-to-firm consistency; however, a thin crust of slightly stronger ash can often be present in the upper $0.5 \mathrm{~m}$ of the pond (Zone 1 in Figure 1).

- A slight increase in ash shear strength would be anticipated, at approximately 1.5 to $2.0 \mathrm{~m}$ in depth across most of the pond's footprint, which is inferred to be due to differing 'ages' i.e. periods of deposition of the Ash Delta deposit over the circa 40 years of operation.

- Generally, the finer (weaker) material associated with the Ash Delta is situated within the southwestern footprint of the pond (Zone 3).

- The phreatic surface (water table) is expected to be located between 0.2 and $1.7 \mathrm{~m}$ below the surface. In most instances, seepage can be observed within charcoal layers, which comprise permeable sand sized particles. 


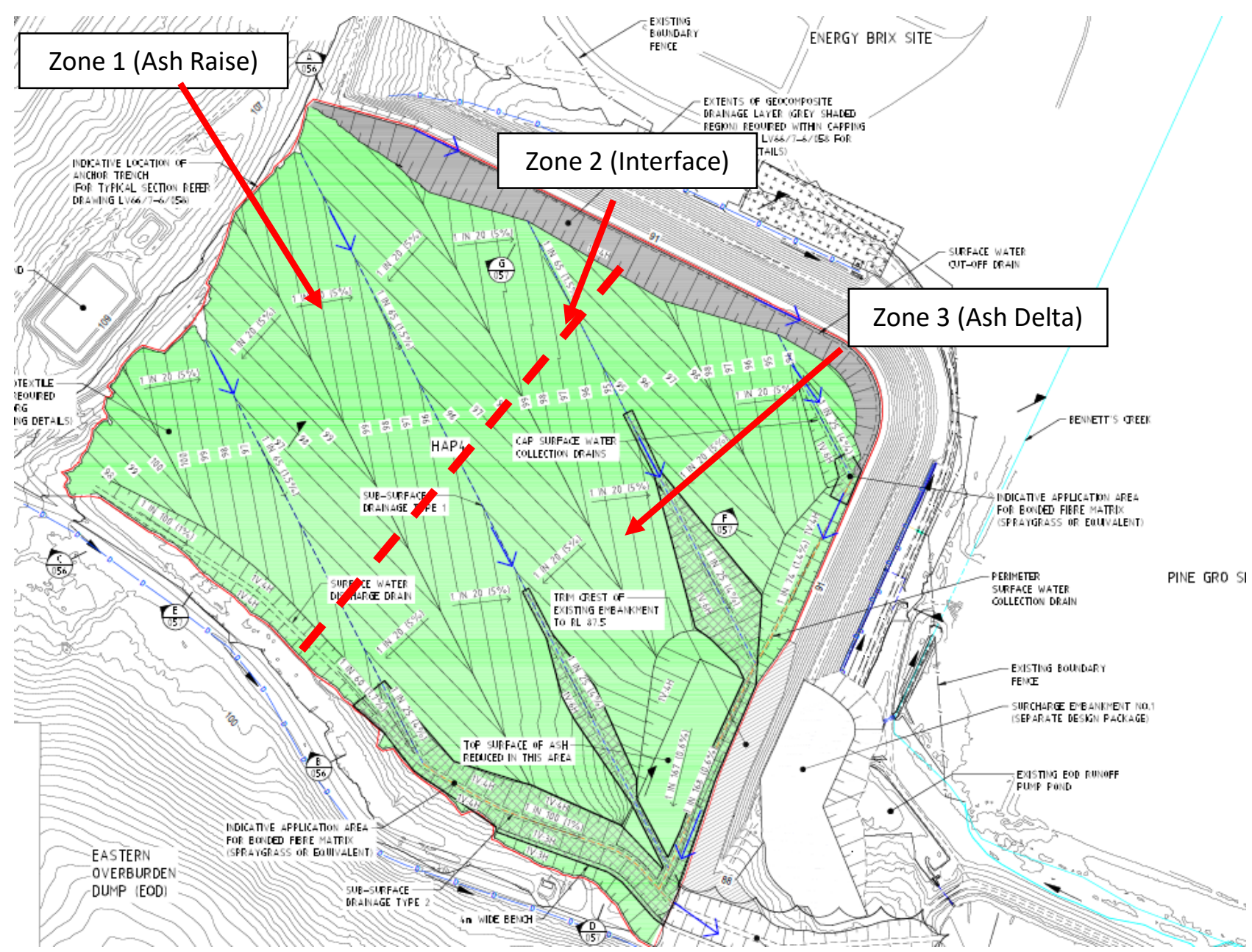

Figure 1 Hazelwood Ash Pond No. 4 (HAP4) ash pond

For the purpose of this study, the ash in HAP4 was divided into three zones (Figure 1):

- Zone 1-Ash Raise Area: the ash in this area is expected to exhibit (comparatively) the least amount of settlement as a result of primary compression. The magnitude of settlement as a result of secondary compression is also anticipated to be relatively low.

- Zone 2 - Interface between Ash Raise and Ash Delta Areas: the ash in this zone is expected to exhibit a moderate amount of settlement (i.e. somewhat greater than Zone 1), as a result of primary compression. The magnitude of settlement as a result of secondary compression is anticipated to be somewhat higher than Zone 1.

- Zone 3-Ash Delta Area: ash in this zone is expected to exhibit the highest amount of settlement comparatively (i.e. greater than Zones 1 and 2) as a result of primary compression, and the magnitude of settlement as a result of secondary compression is also anticipated to be higher than Zones 1 and 2.

\subsection{Challenges associated with conventional geotechnical sampling at HAP4}

Owing to the mechanical characteristics of the ash properties, it is evident that a conventional geotechnical sampling programme is unlikely to provide an accurate representation of in situ conditions and would also render sample recovery challenging. The reasons for this include:

- Trafficability of drilling rigs on the Ash Delta will be challenging due to the mechanical characteristics of the ash. 
- Poor or limited sample recovery due to disturbance induced during the processes of drilling and sampling.

- Handling of recovered ash samples during transport and laboratory preparation would alter sample characteristics before laboratory testing.

Notwithstanding the above challenges, ash samples were sourced and tested via conventional means, and consolidation parameters were interpreted for comparative purposes. It was acknowledged that these properties may not be representative of the ash characteristics within the entire HAP4 footprint across the three material zones.

\section{$5 \quad$ Conceptual settlement estimates}

The consolidation parameters obtained from laboratory testing were utilised to conceptually assess settlements across the HAP4 footprint as a result of the planned earthworks and the capping liner placement. The intent of these estimates was to obtain a basis to compare with subsequent settlements obtained from the trial embankments. Conventional one-dimensional linear consolidation theory after Terzaghi (1943) was utilised. The existing vertical stresses and the anticipated increase in stress under the embankment and/or fill loads were calculated from the individual material layer thicknesses, position of groundwater table and unit weights of the in situ materials, as well as expressions of stress distribution based on elastic soil behaviour. Equation 1 outlines the formulae used to calculate settlement (see Table 1 for a definition of terms), after Terzaghi (1943), noting that there are up to three material layers within HAP4, refer to Figure 2. The settlement ( $p$ ) of the top of the ash layer as a result of the imposition of the vertical stress (fill) $\Delta \sigma_{V}$, is found by summing the contributions of each of the contributory materials (NB: it is assumed that the underlying foundation is 'incompressible'):

$$
\begin{gathered}
p=\sum(\text { stress change } \times \text { stressed length } \times \text { compressibility }) \\
=\Delta \sigma_{V}\left(z_{A} \times m_{v A}+z_{B} \times m_{v B}+z_{C} \times m_{v C}\right)
\end{gathered}
$$

Assume the initial vertical effective stress is at the mid depth of Layer B (Existing Ash):

$$
\sigma_{i B}=\rho_{A} g z_{A}+\rho_{b} g \frac{z_{B}}{2}
$$

Final vertical stress at the mid depth of Layer B:

$$
\sigma_{f B}=\sigma_{i B}+\Delta \sigma_{V}
$$

Average stress level:

$$
\sigma_{a v}=\sigma_{i B}+\frac{\Delta \sigma_{V}}{2}
$$

The settlement:

$$
p=m_{V} \times \Delta \sigma_{V} \times z
$$

See Table 1 for definitions of terms used in Equations 1-5. 
Table $1 \quad$ Definition of terms

\begin{tabular}{|c|c|c|c|}
\hline Term & Symbol & \multicolumn{2}{|l|}{ Definition } \\
\hline Settlement & $p$ & \multicolumn{2}{|c|}{ Downward movement of materials } \\
\hline Vertical stress & $\Delta \sigma_{V}$ & \multicolumn{2}{|c|}{ Contribution of all consolidated material layers } \\
\hline Depth & $z$ & \multicolumn{2}{|c|}{ Stressed length/material layer thickness } \\
\hline Initial effective vertical stress & $\sigma_{i}$ & \multicolumn{2}{|c|}{ Effective stress at the mid of individual layer } \\
\hline Density & $\rho$ & \multicolumn{2}{|l|}{ Unit weight } \\
\hline Final vertical stress & $\sigma_{f}$ & \multicolumn{2}{|c|}{ Effective stress at the mid of individual layer } \\
\hline Average stress & $\sigma_{a v}$ & \multicolumn{2}{|c|}{ Effective stress to determine appropriate compressibility } \\
\hline Compressibility & $m_{V}$ & \multicolumn{2}{|c|}{$\begin{array}{l}\text { Measure of the relative volume change of material as a } \\
\text { response to stress change }\end{array}$} \\
\hline \multicolumn{4}{|c|}{$\Delta \sigma_{V}$} \\
\hline & $z_{A}$ & $\begin{array}{c}\text { New Ash / } \\
\text { Cap }\end{array}$ & $\begin{array}{l}\text { Compressibility } \\
m_{v A}\end{array}$ \\
\hline & $\underline{z_{8}}$ & Old Ash & $m_{v B}$ \\
\hline & $\mathrm{z}_{\varepsilon}$ & OB Dump & $m_{v C}$ \\
\hline
\end{tabular}

Figure 2 Multiple soft ground profile (NB: OB dump is essentially the foundation material and has since been (notably) pre-consolidated owing to placement of 'old ash')

The following primary settlement estimates were calculated across the HAP4 footprint using this same approach (Table 2).

Table 2 Summary of settlement estimates

\begin{tabular}{cc}
\hline Avg. fill height $(\mathrm{m})$ & Settlement $(\mathbf{m m})$ \\
\hline 1.0 to 2.0 & $\sim 300$ \\
3.0 & $\sim 510$ \\
5.0 & $\sim 1,000$ \\
8.0 & $\sim 1,900$ \\
\hline
\end{tabular}

\subsection{Verification of settlement estimates with trial embankments}

To refine the preliminary calculations presented in line with the known variability within the ash associated with HAP4, three trial pads were proposed and constructed at representative locations within the HAP4 footprint as shown in Figure 3. The pad locations were placed in a manner to capture as much of the in situ variability as possible across the three zones. Five settlement plates were installed on each of the three trial pads (Figure 3). The settlement plates serve as markers that can be regularly surveyed to monitor vertical movement. 


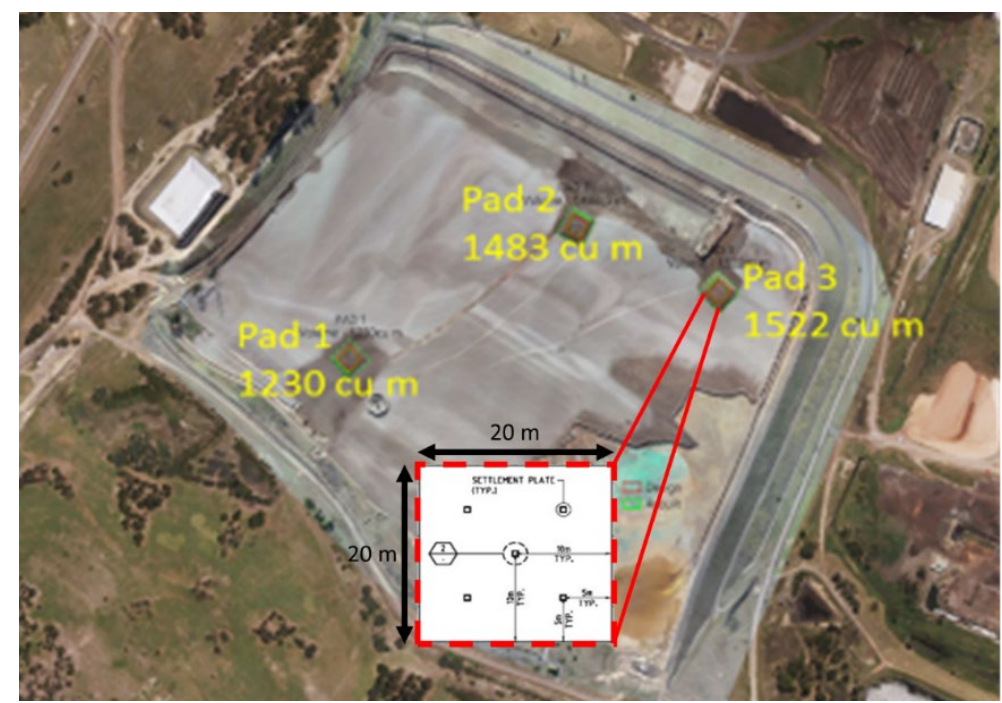

\section{Figure 3 Settlement trial pads}

The pads were installed to the following dimensions:

- $20 \mathrm{~m}$ in width.

- $20 \mathrm{~m}$ in length.

- $2 \mathrm{~m}$ in height.

Settlement was monitored with daily survey readings of the settlement plate prisms taken during the placement of the trial pad fill and continuing for one week after all fill material had been placed. Subsequent readings were taken on a regular basis (fortnightly).

\subsubsection{Interpretation of field data}

The following steps outline the methodology that the authors utilised to interpret the data obtained from surveying the trial embankments, after Asaoka (1978), to verify the preliminary estimates on ultimate primary settlement, $\delta_{100}$, and the corresponding coefficient of compressibility $\left(m_{v}\right)$ for the HAP4 pond, accounting for the variability associated with the respective (three) zones. The survey data was plotted against the elapsed time, as shown in Figure 4:

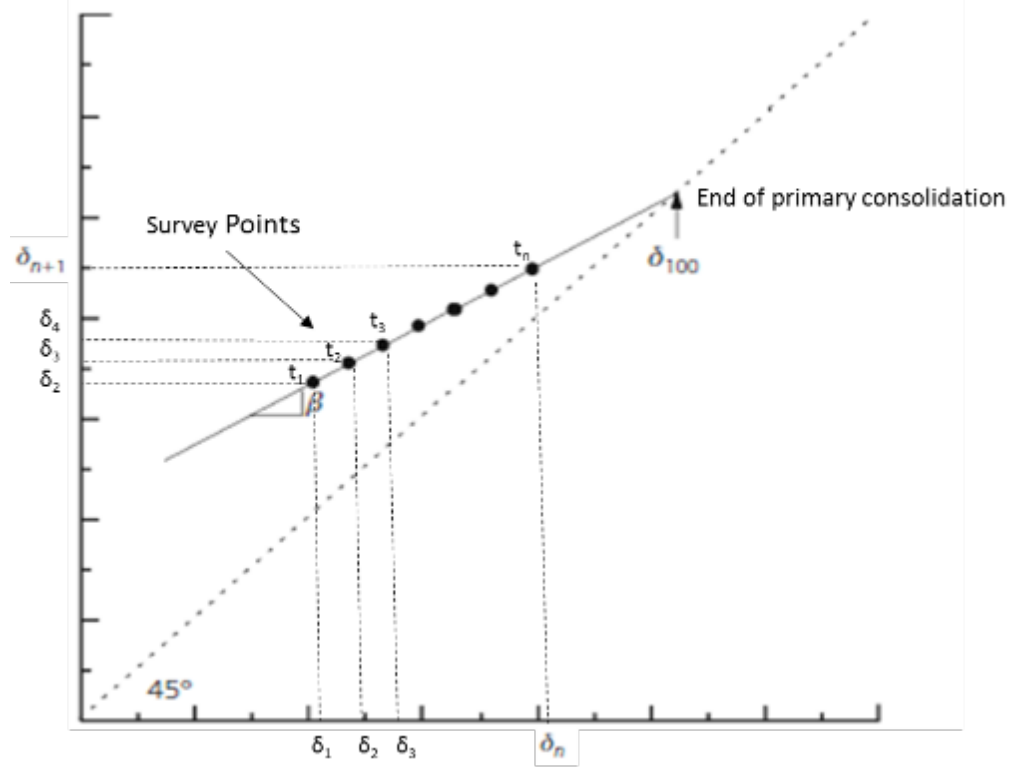

Figure 4 Typical form of Asaoka's chart 
1. Horizontal axis $-\delta_{n}$.

2. Vertical axis $-\delta_{n+1}$, where time intervals are $t_{1}, t_{2}, t_{3}, \ldots t_{n}, t_{n+1}$, such that $t_{n+1}-t_{n}$ is constant.

3. A straight line linear trend, referred to as the $45^{\circ}$ line will be established and extrapolated through the origin.

4. Subsequent survey readings i.e. $\delta_{n+1}$ will be projected (dashed line, depicting a liner trend) to intersect the $45^{\circ}$ line (Figure 4).

5. The point of intersection defines $\delta_{100}$, i.e. the maximum anticipated settlement at primary compression.

6. The value of compressibility coefficient $\left(m_{v}\right)$ can then be back-calculated by substituting $\delta_{100}$ into the one-dimensional consolidation equation after Terzaghi (1943), Equation 1.

Shown across the three charts (Figures 5, 6 and 7) below are combined plots of the survey information for the three different material zones, Ash Delta (Zone 1), the Ash Raise (Zone 3) and the interface (Zone 2) between the two areas. The interpreted $\delta_{100}$ for each of the three areas are summarised in Table 3 along with the settlement that had occurred to date (i.e. at the time of interpretation).

Table 3 Summary of settlement observations and interpretations

\begin{tabular}{lcccc}
\hline Trial pad ID & $\begin{array}{c}\text { Settlement to } \\
\mathbf{1 5} \text { Oct } \mathbf{2 0 1 8}(\boldsymbol{\Sigma} \boldsymbol{\delta})(\mathbf{m m})\end{array}$ & $\boldsymbol{\delta}_{\mathbf{1 0 0}}(\mathbf{m m})$ & $\begin{array}{c}\text { Theoretical remaining } \\
\text { settlement }(\mathbf{m m}) \text { after Asoka }\end{array}$ & $\begin{array}{c}\text { Degree of } \\
\text { consolidation }\end{array}$ \\
\hline Ash Delta (Pad 3) & 70 & 102 & 32 & $68 \%$ \\
Interface (Pad 2) & 95 & 110 & 15 & $86 \%$ \\
Ash Raise (Pad 1) & 135 & 140 & 5 & $96 \%$ \\
\hline
\end{tabular}

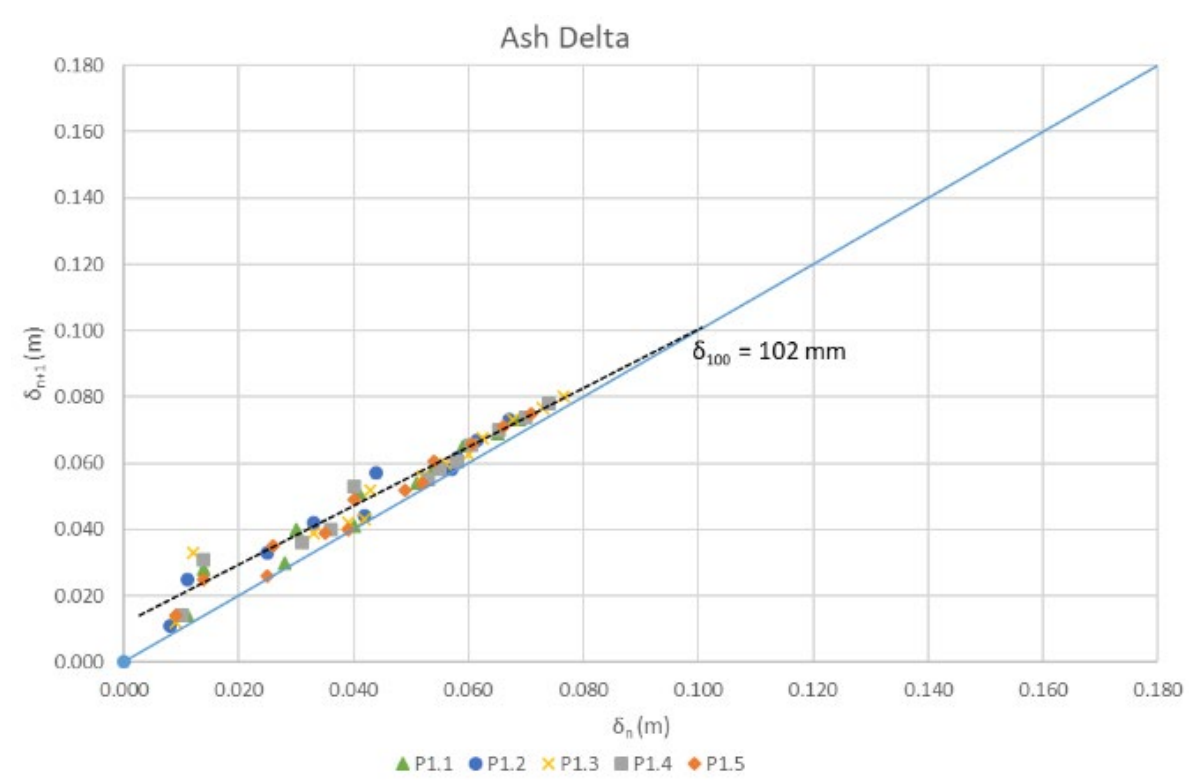

Figure 5 Ash Delta layer settlement observations ( $\mathrm{Pad} 3)$ 


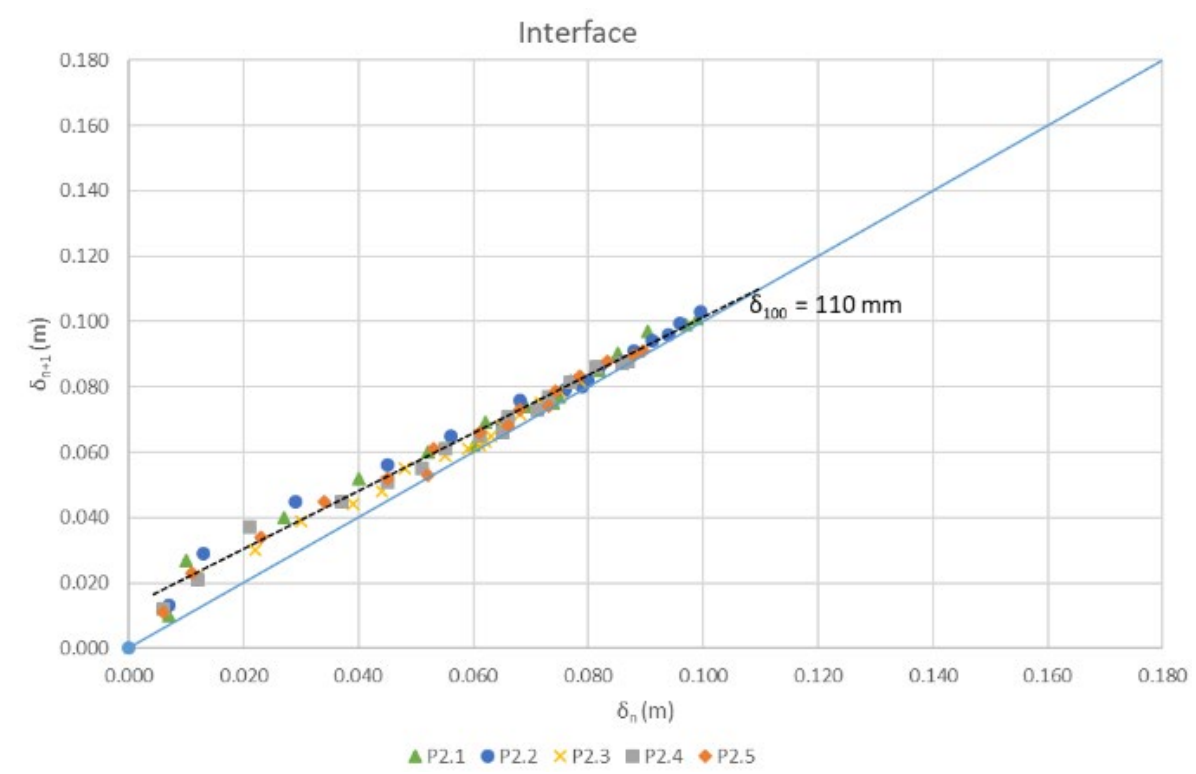

Figure 6 Interface Layer settlement observations (Pad 2)

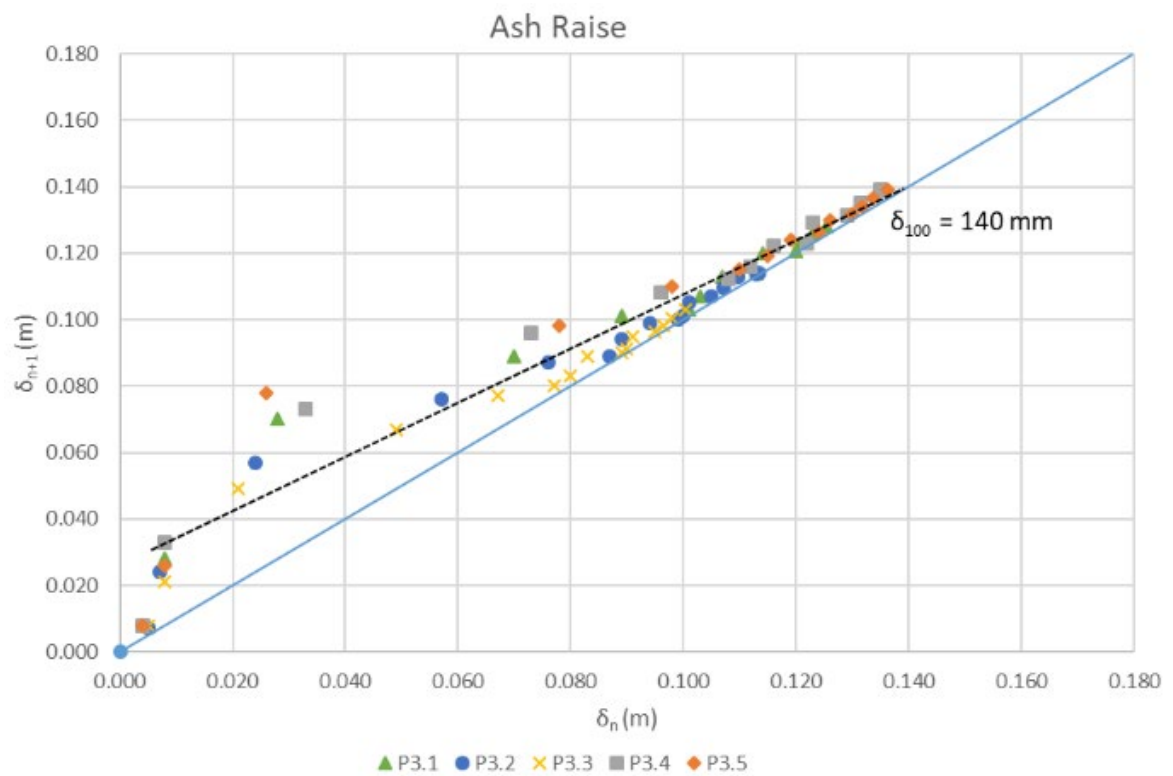

Figure 7 Ash Raise layer settlement observations (Pad 1)

The authors applied the following approach in back-calculating the compression coefficients $\left(m_{v}\right)$ across each of the three trial embankment areas, noting that the literature indicates that Asaoka's method for interpreting settlement magnitudes $\left(\delta_{100}\right)$ as a result of primary compression, are considered comparatively more reliable when $80 \%$ of primary compression (or greater) has been achieved. For instance, a prediction of total primary settlement magnitudes made on an area that has achieved $60 \%$ of primary compression would be comparatively less reliable that one made within an area that has achieved say $90 \%$ of primary compression:

- Based on this it is likely that the $\delta_{100}$ values interpreted for Pads 2 and 3 are likely to be more reliable than that calculated for Pad 1 , as Pads 2 and 3 have achieved $>80 \%$ of primary compression.

- The total magnitude of predicted settlement $\left(\delta_{100}\right)$ at primary compression was utilised to back calculate the compressibility coefficient across each of the three trial embankments, using Equation 1. 
- The back-calculated compressibility coefficients were attributed with a qualitative 'reliability' rating (either high, medium or low) based on the degree of primary compression achieved thus, based on Asaoka's theory (1978):

- High - greater than $90 \%$ of primary compression achieved.

- Medium - $>80 \%$ of primary compression achieved, but $<90 \%$.

- Low - less than $80 \%$ of primary compression achieved.

- Subsequent to these steps, adjustments were made to the calculated settlements based on their reliability ratings which are explained further herein.

\subsubsection{Back calculation of consolidation parameters}

The back-calculated compressibility coefficients are tabulated in Table 4.

Table 4 Back-calculated compressibility coefficients

\begin{tabular}{lccccc}
\hline Area & $\begin{array}{c}\text { Fill height } \\
(\mathbf{m})\end{array}$ & $\begin{array}{c}\boldsymbol{\delta}_{100} \\
(\mathbf{m m})\end{array}$ & $\begin{array}{c}\text { Degree of } \\
\text { consolidation }\end{array}$ & Compressibility, $\mathbf{~ m v ~}\left(\mathbf{m}^{2} / \mathbf{k N}\right)$ & $\begin{array}{c}\text { Assigned } \\
\text { reliability }\end{array}$ \\
\hline Ash Delta & 2.0 & 102 & $68 \%$ & $0.102 \div(2 \times 153.9)=3.31 \times 10^{-4}$ & Low \\
$\begin{array}{l}\text { Ash Raise } \\
\begin{array}{l}\text { Interface (Ash } \\
\text { Raise/Delta) }\end{array}\end{array}$ & 2.0 & 110 & $86 \%$ & $0.110 \div(2 \times 153.9)=3.57 \times 10^{-4}$ & Medium to high \\
\hline
\end{tabular}

The compressibility coefficient $\left(\mathrm{m}_{\mathrm{v}}\right)$ along the interface of the Ash Raise and the Delta is $4.55 \times 10^{-4}\left(\mathrm{~m}^{2} / \mathrm{kN}\right)$. This value has a high reliability associated with it and can be considered reasonable to utilise in the calculation of settlements within the Ash Raise/Delta interface. The compressibility coefficient $\left(m_{v}\right)$ for the Ash Raise is $3.57 \times 10^{-4}$. This value has a medium reliability associated with it but nonetheless can be considered reasonable to utilise in the calculation of settlements within the Ash Raise. The compressibility coefficient $(\mathrm{mv})$ for the Ash Delta is $3.31 \times 10^{-4}$. This value has a low reliability associated with it and may therefore have a higher component of secondary compression associated with it, which can be difficult to establish using Asaoka's approach.

\subsection{Determination of design settlements from primary compression}

Based on the settlement results obtained over the circa 12 weeks and the back-calculated compression coefficients, the below tabulated primary settlement thresholds were calculated for the Ash Raise and the Raise-Delta interface (Table 5). The expected primary settlement thresholds in the Ash Delta area remains uncertain. This gap would need to be addressed to ensure that the final cap is appropriately designed to meet Landfill BPEM requirements.

Table 5 Summary of primary settlement magnitudes

\begin{tabular}{cccc}
\hline $\begin{array}{c}\text { Fill height } \\
(\mathbf{m})\end{array}$ & $\begin{array}{c}\text { Settlement Ash } \\
\text { Raise }(\mathbf{m m})\end{array}$ & $\begin{array}{c}\text { Settlement Interface } \\
\text { Ash Raise-Delta }(\mathbf{m m})\end{array}$ & $\begin{array}{c}\text { Ash Delta } \\
(\mathbf{m m})\end{array}$ \\
\hline 1.0 & 49 & 63 & $?$ \\
2.0 & 110 & 140 & $?$ \\
3.0 & 182 & 229 & $?$ \\
5.0 & 359 & 457 & $?$ \\
8.0 & 689 & 868 & $?$ \\
\hline
\end{tabular}




\subsection{Discussion of findings thus far}

Based on the observations from the trial embankments within the Ash Raise and the Ash Delta interface, it has been found that the settlement predictions made from the trial pads appear to significantly overestimate the predicted thresholds of primary settlement originally estimated. This can have a significant bearing on the commercial aspects of the projects and the performance of the capping liner for these reasons:

1. Where settlement is expected, provision needs to be made within the earthworks plan to 'top up' the areas that have settled to the designed levels.

2. Accordingly, poor predictions of settlements can lead to over or underestimated construction costs. In the case of HAP4 there was a potential to significantly overestimate the earthworks volumes within the Ash Raise area.

3. Furthermore, as the capping liner has been designed to accommodate settlement to achieve particular (minimum) grades to facilitate water-shedding in the long-term. Where predicted settlements fail to occur the camber on the liner may not achieve the required grade, thereby resulting in water ponding atop the liner potentially degrading it prior to its serviceable life.

In addition to these points there appears to be a degree of uncertainty around the predictions associated with the anticipated primary settlements within the Ash Delta. The observations made from the trial embankments within this area, indicate that there is a potential for a degree of secondary compression. For the same reasons outlined, it is important that these are understood and quantified, along with the anticipated timing, so as to ensure that the construction can be suitably staged, and liner installation within this area delayed so as to accommodate the effects of secondary compression.

Owing to the points mentioned and the confidence required in the liner being able to accommodate the primary and secondary compression within the Ash Delta, it was deemed necessary to continue to monitor the trial embankment within this area to assess its achievement of primary consolidation. The findings so far have shed some light of the consolidation behaviour of the Hazelwood Power Station ash as follows:

1. When using laboratory assessments of consolidation parameters to calculate settlements within the Ash Raise there appears to be a potential to overestimate the magnitudes of settlement. This could be as a result of:

a. The challenges associated with the retrieval, transportation and handling of such samples, as outlined previously. i.e. whereby the remoulding of the samples in the laboratory may alter the mechanical characteristics of the sample in comparison to its native form (i.e. in comparison to in situ conditions).

b. Additionally, it is possible that a component of the predicted primary settlement in the Ash Raise occurs summarily during the process of earth working and thus may not be observed as part of the primary consolidation phase.

2. The observations from the trial embankments within the Ash Raise gives rise to the following understanding in relation to the timing and associated magnitudes of settlement:

a. Immediate settlement was in the order of one-third of the total estimated settlement within the Ash Raise (data not shown), and occurred within 1 week of the completion of the earthworks.

b. $90 \%$ of consolidation occurred within 10 to 15 weeks of construction within the Ash Raise.

3. The deposition chronology and mechanical characteristics of the ash within the delta renders it susceptible to protracted periods to reach primary consolidation, and secondary compression is a crucial consideration. 


\subsection{Approach to addressing 'gaps' and the finalisation design with confidence}

The gaps and challenges primarily associated with the consolidation behaviour of the Ash Delta, were deemed crucial. These required addressing to put forward a final (robust) capping design that can accommodate and tolerate the anticipated settlements and remain serviceable.

The following measures were taken:

- Further representative laboratory test results were sourced and the secondary compression behaviour was assessed.

- The embankment in the delta was subjected to continued monitoring to assess its achievement of primary consolidation.

This resulted in the following estimates for total settlement, with the primary compression component being in the order of 150 to $200 \mathrm{~mm}$.

Table 6 Summary of estimated total settlement

\begin{tabular}{cc|}
\hline Fill height $(\mathbf{m})$ & Ash Delta $(\mathbf{m m})$ \\
\hline 1.0 & 298 \\
2.0 & 468 \\
3.0 & 683 \\
5.0 & 1,194 \\
8.0 & 2,140 \\
\hline
\end{tabular}

The increased confidence associated with the consolidation behaviour (i.e. magnitudes and timing of the power station ash within HAP4) enabled the liner and earthworks levels to be designed with certainty to accommodate the behaviour of the three materials zones and comply with the requirements outlined in the EPA Landfill BPEM. As it was known that the magnitudes of settlement within the Ash Delta can be higher and would include a significant component of secondary compression, fill heights in this area were minimised to reduce the magnitudes of settlement. Additionally, a camber was also designed into the geometry of the capping liner to accommodate the effects of secondary compression.

\section{Conclusion}

The results outlined in this paper has highlighted the challenges associated with designing and installing a capping layer on brown coal ash ponds where little is understood of its consolidation behaviour. Based on the work undertaken by the authors with the support of the Hazelwood mine, an approach was developed and successfully tested with the benefit of onsite experimentation to ensure that the in situ variability and settlement performance can be quantified and incorporated to ensure a EPA (Landfill BPEM) compliant capping can be suitably designed and installed to contain the HAP4 ash pond at ENGIE's Hazelwood Mine. The authors are of the opinion this body of information along with the approach applied will prove beneficial to other brown coal mines within the Latrobe Valley that eventually transition to closure.

\section{Acknowledgement}

The authors would like to thank ENGIE for providing the opportunity to publish this paper and in particular Mr Brad Marvin, ENGIE Project Manager for his guidance during the planning and execution of this work package, Mr Slavko Kacavenda of GHD Pty Ltd for his technical guidance during the course of this project and Ms Rebecca Turnbull of GHD Pty Ltd for editing this paper. 


\section{References}

Asaoka, A 1978, 'Observational procedure of settlement prediction', Soils and Foundations, vol. 18, no. 4, pp. 87-101.

Duncan, JM 1993, 'Limitations of conventional analysis of consolidation settlement', Journal of Geotechnical Engineering, vol. 119, pp. 1333-1359.

Environmental Protection Authority (EPA) Victoria 2015, Best Practice Environmental Management: Siting, design, operation and rehabilitation of landfills, Publication 788.3 (Landfill BPEM), August 2015.

Mudd, GM, Chakrabarti, S \& Kodikara, J 2006, 'Evaluation of engineering properties for the use of leached brown coal ash in soil covers', Journal of Hazardous Materials, vol. 139, iss. 3, pp. 409-412.

Terzaghi, K 1943, Theoretical Soil Mechanics, John Wiley \& Sons, New York, 510 p. 